\author{
Dinna N. Cruz \\ Massimo de Cal \\ Francesco Garzotto \\ Mark A. Perazella \\ Paolo Lentini \\ Valentina Corradi \\ Pasquale Piccinni \\ Claudio Ronco
}

\section{Plasma neutrophil gelatinase-associated lipocalin is an early biomarker for acute kidney injury in an adult ICU population}

Received: 28 May 2009

Accepted: 18 September 2009

Published online: 3 December 2009

(C) The Author(s) 2009. This article is published with open access at

Springerlink.com

This article is discussed in the editorial available at:

doi:10.1007/s00134-009-1733-8.

Electronic supplementary material The online version of this article (doi:10.1007/s00134-009-1711-1) contains supplementary material, which is available to authorized users.

D. N. Cruz ( $)$ M. de Cal · F. Garzotto · P. Lentini · V. Corradi - C. Ronco Department of Nephrology,

Dialysis and Transplantation,

St Bortolo Hospital, Viale Rodolfi 37, 36100 Vicenza, Italy

e-mail: dinnacruzmd@yahoo.com

Tel.: +39-0444-753650

Fax: +39-0444-753949

C. Ronco

e-mail: cronco@goldnet.it

D. N. Cruz - M. de Cal - F. Garzotto •

C. Ronco

International Renal Research Institute

Vicenza (IRRIV), Vicenza, Italy
M. A. Perazella

Section of Nephrology, Yale University

School of Medicine, New Haven, CT, USA

P. Piccinni

Department of Intensive Care,

San Bortolo Hospital, Vicenza, Italy

Abstract Purpose: Neutrophil gelatinase-associated lipocalin (NGAL) is a useful marker for acute kidney injury (AKI), particularly when the timing of renal insult is known. However, its performance in an adult critical care setting has not been well described. We performed this study to estimate the diagnostic accuracy of plasma NGAL for early detection of AKI and need for renal replacement therapy (RRT) in an adult intensive care unit (ICU). Methods: We enrolled 307 consecutive adult patients admitted to a general medical-surgical ICU; 301 were included in the final analysis. Serial blood samples were analyzed for plasma NGAL using a standardized clinical platform. The primary outcome was AKI, defined as an increase in creatinine of at least 50\% from baseline or a reduction in urine output to $<0.5 \mathrm{ml} / \mathrm{kg} / \mathrm{h}$ for $>6 \mathrm{~h}$. Results: Of 301 patients, 133 (44\%) had AKI during their ICU stay. Plasma NGAL was a good diagnostic marker for AKI development within the next $48 \mathrm{~h}$ (area under ROC 0.78 , 95\% CI 0.65-0.90), and for RRT use (area under ROC $0.82,95 \%$ CI 0.70 0.95). Peak plasma NGAL concentrations increased with worsening AKI severity $(R=0.554$, $P<0.001)$. Conclusions: Plasma NGAL is a useful early marker for AKI in a heterogeneous adult ICU population, in which the timing of renal insult is largely unknown. It allows the diagnosis of AKI up to $48 \mathrm{~h}$ prior to a clinical diagnosis based on AKI consensus definitions. Additionally, it predicts need for RRT and correlates with AKI severity. Early identification of high risk patients may allow potentially beneficial therapies to be initiated early in the disease process before irreversible injury occurs.

Keywords Acute kidney injury · Acute renal failure - Adult . Biomarker - Diagnostic accuracy . Intensive care unit - NGAL . Renal replacement therapy . Sensitivity $\cdot$ Specificity 


\section{Introduction}

Acute kidney injury (AKI) is a common and serious process that occurs in a wide variety of clinical settings. Lack of early AKI biomarkers has impeded timely interventions to ameliorate the downstream effects of AKI and has hindered further clinical investigation of therapies that are effective in animal studies.

Neutrophil gelatinase-associated lipocalin (NGAL) is a promising biomarker for early detection of AKI [1]. Several studies have shown serum and urine NGAL to be useful early markers for AKI, particularly when the timing of the renal insult is known, such as post-cardiac surgery and radiocontrast exposure [2, 3]. However, its performance in a more general intensive care unit (ICU) setting has not been as well described. Urine NGAL was found to be a useful early marker for development of AKI in pediatric ICU patients (AuROC 0.78) [4], whereas serum NGAL was a highly sensitive but nonspecific predictor of AKI in critically ill children with septic shock (AuROC 0.68) [5]. We hypothesized that plasma NGAL would also be an early biomarker of AKI in an unselected population of critically ill adults.

The main objective of the study was to estimate the diagnostic accuracy of plasma NGAL (pNGAL) in an adult general ICU for the early detection of AKI. Additional objectives were to evaluate pNGAL as a biomarker for renal replacement therapy (RRT) use and mortality in the ICU, as well as to explore the relationship between pNGAL, AKI severity and overall severity of illness.

\section{Concise methods}

Study design and setting

This was a prospective observational study of consecutive adult patients admitted to a general ICU from January to July 2007. Patients were enrolled within $24 \mathrm{~h}$ of ICU admission; informed consent was obtained from the patient surrogate. Detailed methods are described in ESM File 1.

\section{Clinical and laboratory data collection}

Multiple data elements were collected for each patient and included demographics, co-morbidities, admission diagnosis, severity of illness scores, RRT provision and ICU mortality. Clinical charts were reviewed for a history of exposure to potential nephrotoxins and use of diuretics in the ICU.

Laboratory data were measured daily as part of routine patient care. Blood samples for NGAL were collected daily from ICU admission for up to 4 days. Reasons for not collecting blood samples for NGAL for all 4 days included death, ICU discharge and ongoing RRT.

\section{Definition of acute kidney injury}

AKI was defined using the creatinine and urine output criteria of the RIFLE (Risk-Injury-Failure-Loss-Endstage renal disease) classification [6]. Baseline renal function was defined as the lowest known SCr value during the preceding 3 months. For patients without known prior SCr, the baseline serum creatinine was estimated by solving the MDRD equation [6, 7].

Patients were classified daily using the RIFLE criteria. RIFLE-initial refers to their RIFLE class on the first day of AKI. RIFLE-max refers to the worst RIFLE class reached by a patient during his ICU stay. For AKI patients, renal status at ICU discharge was defined as follows: Complete recovery if discharge $\mathrm{SCr}$ was $<150 \%$ of baseline; partial recovery if discharge $\mathrm{SCr}$ was 150 $199 \%$ of baseline; non-recovery if discharge $\mathrm{SCr}$ was $\geq 200 \%$ of baseline or still receiving RRT.

\section{Sampling and measurement of NGAL}

Blood samples for NGAL were collected daily, and samples were processed within $1 \mathrm{~h}$ from time of collection. We centrifuged samples at $3,500 \times g$ for $10 \mathrm{~min}$ and stored the supernatant in equal volumes at $-80^{\circ} \mathrm{C}$ until measurement. Plasma NGAL (pNGAL) was measured using the Triage ${ }^{\circledR}$ NGAL Test (Biosite Inc, San Diego, CA), a point-of-care, fluorescence-detected immunoassay. We measured pNGAL in 873 blood samples (median 3 samples/patient). Laboratory investigators were blinded to sample sources and clinical information until the end of the study. For comparative purposes, pNGAL was measured in banked anti-coagulated plasma of apparently healthy donors $(n=120)$.

\section{Statistical analysis}

Continuous variables are expressed as mean \pm standard deviation or median (interquartile range) and compared between any two groups using t-test or the Mann-Whitney $U$ test, and among three or more groups using analysis of variance (general linear models with adjustment for multiple comparisons) or the Kruskal-Wallis test, where appropriate. Categorical variables are expressed as proportions and compared with the chi-square test or Fisher's exact test.

Diagnostic characteristics of pNGAL were evaluated with receiver-operating characteristic (ROC) curves. The primary outcome of interest was prediction of AKI. Based on prior studies $[4,8]$, for our primary analysis we defined an event as AKI occurring within $48 \mathrm{~h}$ of first pNGAL 
measurement. Secondary outcomes included RRT use, ICU mortality, and their composite endpoint.

After seeing the relationship between pNGAL and these scores, we also performed a post hoc covariate analysis using multiple logistic regression to determine the independent predictive ability of the first pNGAL for AKI within $48 \mathrm{~h}$. The following covariates were considered: age, APACHE, SAPS and SOFA scores. For all analyses, two-tailed $P$ values $<0.05$ were considered significant. Statistical analyses were conducted using SPSS 15 (SPSS Inc., Chicago, IL) and MATLAB 7.5.0 (The MathWorks, Inc., Natick, MA).

\section{Results}

Patient demographics

We enrolled 307 consecutive incident patients to an adult medical-surgical ICU. We excluded 5 patients with endstage renal disease on chronic RRT and 1 patient for uninterpretable NGAL result, leaving 301 patients for analysis. Patient characteristics are shown in Table 1 . Two hundred twenty patients $\left(73 \%\right.$ ) had an eGFR $<90 \mathrm{ml} / \mathrm{min} / 1.73 \mathrm{~m}^{2}$, of whom $20(6.6 \%)$ had an eGFR $<60 \mathrm{ml} / \mathrm{min} / 1.73 \mathrm{~m}^{2}$. The most common diagnostic categories for ICU admission were Neurologic, Respiratory, Cardiovascular, Trauma and Gastrointestinal, accounting for $89 \%$ of admissions. Median ICU length of stay was 7 days (IQR 3,13). Crude ICU and hospital mortalities were 17.3 and $25.5 \%$, respectively, and $58(19.3 \%)$ patients met the composite RRT or ICU death endpoint.

\section{Acute kidney injury}

Of 301 patients, $133(44 \%)$ had AKI during their ICU stay; 90 patients had AKI within $24 \mathrm{~h}$ of ICU admission, while 43 developed it later during their ICU stay (median 3rd day, IQR 2-5) (see ESM File 1, ESM Table 1). RIFLE-initial class was Risk in 92 patients (30.6\%),

Table 1 Characteristics of the cohort by presence or absence of acute kidney injury (AKI)

\begin{tabular}{|c|c|c|c|c|}
\hline & All & Non-AKI & AKI & $P$ \\
\hline$N$ & 301 & 168 & 133 & \\
\hline Male sex $(\%)$ & $207(68.8)$ & $113(67.3)$ & $94(70.7)$ & 0.53 \\
\hline Age (years) & $64(45,74)$ & $58(37,70.5)$ & $69(61,76.5)$ & $<0.001$ \\
\hline Body weight (kg) & $76.5 \pm 12.3$ & $75.6 \pm 13.1$ & $77.7 \pm 11.2$ & 0.16 \\
\hline Baseline creatinine $(\mathrm{mg} / \mathrm{dl})$ & $0.9(0.8,1.0)$ & $0.9(0.8,1.0)$ & $1.0(0.8,1.0)$ & 0.035 \\
\hline Diabetes $(\%)$ & 47 (15.6) & $16(9.5)$ & $31(23.3)$ & 0.001 \\
\hline Chronic kidney disease $(\%)$ & $20(6.6)$ & $8(4.8)$ & $12(9.0)$ & 0.16 \\
\hline \multicolumn{5}{|l|}{ Patient type } \\
\hline Medical (\%) & $164(54.6)$ & $85(50.6)$ & $79(59.4)$ & 0.13 \\
\hline Elective surgery $(\%)$ & $25(8.3)$ & $14(8.3)$ & $11(8.3)$ & 1.00 \\
\hline Emergency surgery $(\%)$ & $112(37.1)$ & $69(41.1)$ & $43(32.3)$ & 0.15 \\
\hline \multicolumn{5}{|l|}{ Admission diagnosis } \\
\hline Neurologic $(\%)$ & $90(29.9)$ & $66(39.3)$ & $24(18)$ & $<0.001$ \\
\hline Respiratory (\%) & $57(18.9)$ & $24(14.3)$ & $33(24.8)$ & 0.026 \\
\hline Cardiovascular (\%) & $51(16.9)$ & $23(13.7)$ & $28(21.1)$ & 0.12 \\
\hline Trauma (\%) & $49(16.3)$ & $31(18.5)$ & $18(13.5)$ & 0.27 \\
\hline Gastrointestinal (\%) & $22(7.3)$ & $10(6.0)$ & $12(9.0)$ & 0.374 \\
\hline \multicolumn{5}{|l|}{ ICU admission } \\
\hline SAPS II & $41 \pm 15$ & $38 \pm 15$ & $45 \pm 14$ & $<0.001$ \\
\hline APACHE II & $18 \pm 7$ & $16 \pm 7$ & $20 \pm 7$ & $<0.001$ \\
\hline SOFA & $5(5,7)$ & $5(4,6)$ & $5(5,8)$ & $<0.001$ \\
\hline Creatinine $(\mathrm{mg} / \mathrm{dl})$ & $1.0(0.8,1.4)$ & $0.9(0.8,1.1)$ & $1.3(1.0,2.0)$ & $<0.001$ \\
\hline Plasma NGAL (ng/ml) & $117.0(68.2,213.8)$ & $82.0(49.7,134.1)$ & $185(95.4,403.5)$ & $<0.001$ \\
\hline Potential nephrotoxins (\%) & 38 (12.6) & $15(8.9)$ & $23(17.3)$ & 0.036 \\
\hline Use of vasopressors (\%) & $102(33.9)$ & $35(20.8)$ & $67(50.4)$ & $<0.001$ \\
\hline \multicolumn{5}{|l|}{ ICU course } \\
\hline Diuretics $(\%)$ & $157(52.2)$ & $65(38.7)$ & $92(69.2)$ & $<0.001$ \\
\hline Sepsis $(\%)$ & $115(38.2)$ & $49(29.2)$ & $66(49.6)$ & $<0.001$ \\
\hline \multicolumn{5}{|l|}{ Outcome } \\
\hline ICU mortality (\%) & $52(17.3)$ & $15(8.9)$ & $37(27.8)$ & $<0.001$ \\
\hline RRT or ICU mortality (\%) & $58(19.3)$ & $15(8.9)$ & $43(32.3)$ & $<0.001$ \\
\hline ICU length of stay (days) & $7(3,13)$ & $5(3,10)$ & $9(5,15)$ & $<0.001$ \\
\hline ICU LOS: survivors (days) & $7(3,13)$ & $6(3,10.5)$ & $9(5,15.8)$ & $<0.001$ \\
\hline ICU LOS: nonsurvivors (days) & $7(4,13)$ & $5(2,7)$ & $9(4,16)$ & 0.019 \\
\hline
\end{tabular}

Body weight, SAPS II and APACHE II are expressed as mean \pm standard deviation. All other continuous variables are expressed as median (IQR) 
Injury in 17 (5.6\%) and Failure in 24 (8.0\%). Progression of AKI to a worse RIFLE class was seen in 37 patients (28\% of AKI patients). The maximum RIFLE class reached (RIFLE-max) was Risk in 57 patients (18.9\%), Injury in $42(14 \%)$ and Failure in $34(11.3 \%)$. Fifteen patients $(5 \%)$ were treated with RRT in the ICU. AKI patients were older, less likely to have a neurologic diagnosis and more likely to have a respiratory diagnosis on ICU admission, had higher APACHE II, SAPS II and SOFA scores, and higher baseline and admission SCr values. They were more likely to have had exposure to potential nephrotoxins prior to their ICU admission, and to have received diuretics during their ICU stay. Sixty-six AKI patients $(49.6 \%)$ fulfilled criteria for sepsis during their ICU stay, compared to 49 (29.2\%) of non-AKI patients $(P<0.001)$, whereas 83 AKI patients $(62.4 \%)$ fulfilled criteria for SIRS or sepsis during their ICU stay, as compared to $80(47.6 \%)$ of non-AKI patients $(P=0.014)$.

AKI patients had a higher crude ICU mortality $(27.8 \%$ vs. non-AKI $8.9 \%, P<0.001)$ and longer ICU length of stay [median 9 days vs. 5 days (non-AKI), $P<0.001$ ]. Sixty-four patients (48\% of AKI patients) had complete recovery of renal function, with a $\mathrm{SCr}$ at time of ICU discharge that was $<150 \%$ of baseline; an additional 27 AKI patients (20\%) had partial renal recovery.

\section{NGAL in AKI and non-AKI}

The median first pNGAL in the study population was 117.0 (IQR 68.2, 213.8) ng/ml, which was significantly higher than 61.2 (IQR $60.0-78.5) \mathrm{ng} / \mathrm{ml}$ observed in apparently healthy adults $(P<0.001)$. Even ICU patients without AKI had significantly higher pNGAL levels $(82.0 \mathrm{ng} / \mathrm{ml}, \mathrm{IQR} 49.7,134.1)$ compared to healthy adults $(P=0.004)$. A summary of first pNGAL values in the study population is shown in Fig. 1. Compared to non-

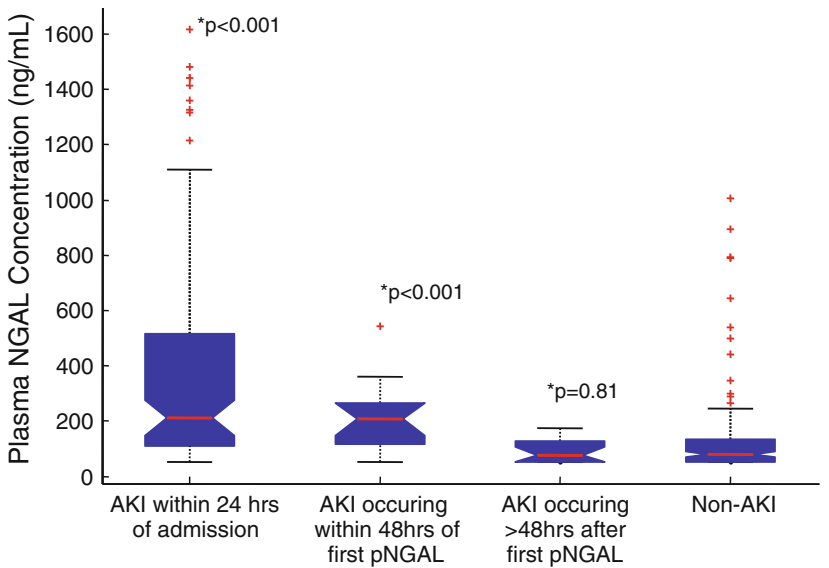

Fig. 1 Plasma NGAL values by timing of AKI. Values represent first plasma NGAL specimen. ${ }^{*} P$-value refers to comparison with Non-AKI group
AKI patients, pNGAL values were higher among patients who already had AKI at the time of first pNGAL measurement $(213.7 \mathrm{ng} / \mathrm{ml}$, IQR $110.4,553.3 ; P<0.001$ vs. non-AKI), as well as in patients who developed AKI within $48 \mathrm{~h}(209.0 \mathrm{ng} / \mathrm{ml}$, IQR 101.4, 268.2; $P<0.001$ vs. non-AKI). Patients who developed AKI within $24 \mathrm{~h}$ had a median pNGAL of $225.3 \mathrm{ng} / \mathrm{ml}$ (IQR 163.0, 279.5; $P<0.001$ vs. non-AKI), whereas those who developed it within $24-48 \mathrm{~h}$ had a median pNGAL of $140.7 \mathrm{ng} / \mathrm{ml}$ (IQR 95.8, 218.3; $P=0.13$ vs. non-AKI). In contrast, patients who developed AKI after $>48 \mathrm{~h}$ had pNGAL similar to non-AKI patients $(79.8 \mathrm{ng} / \mathrm{ml}$, IQR 52, 143.9; $P=0.81$ ). The proportion of AKI patients was higher with progressive quartiles of first pNGAL measurement (see ESM file 1, ESM Fig. $1, P<0.001$ ).

A graphical representation of the kinetics of pNGAL is shown in Fig. 2. For AKI patients, the $X$-axis shows AKI Day $[4,8]$. AKI Day 0 is the day in which the diagnosis of AKI was made based on the RIFLE criteria. One day prior to AKI, pNGAL was higher in these patients compared to non-AKI, and increased further on AKI Day 0. Patients who never developed AKI had comparatively lower pNGAL values without notable variation during the study period $(P<0.001$ at each time point).

NGAL and prediction of clinical outcome

Performance characteristics of pNGAL for prediction of AKI and secondary outcomes are shown in Fig. 3 and Table 2. Plasma NGAL was a good diagnostic marker for

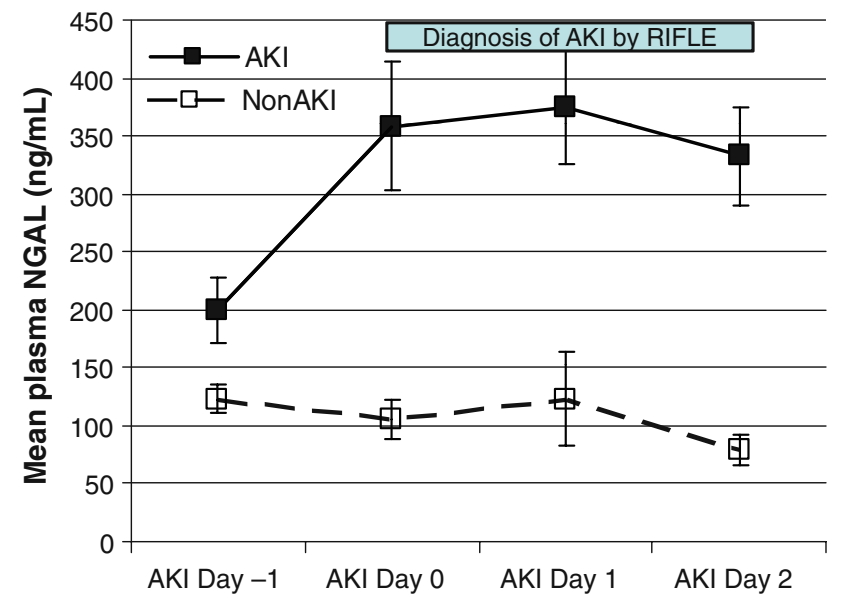

Fig. 2 Mean plasmaNGAL concentrations at various timepoints in patients with (solid squares) and without (open squares) acute kidney injury (AKI). For AKI patients, the $X$-axis shows AKI Day. AKI Day 0 is the day in which the diagnosis of AKI was first made based on the RIFLE criteria. The green bar indicates the days when there is AKI. For non-AKI patients, the $X$-axis represents consecutive NGAL collection days. Patients who never developed AKI had comparatively lower plasma NGAL values without notable variation $(P<0.001$ at each time point). Error bars are SEM 


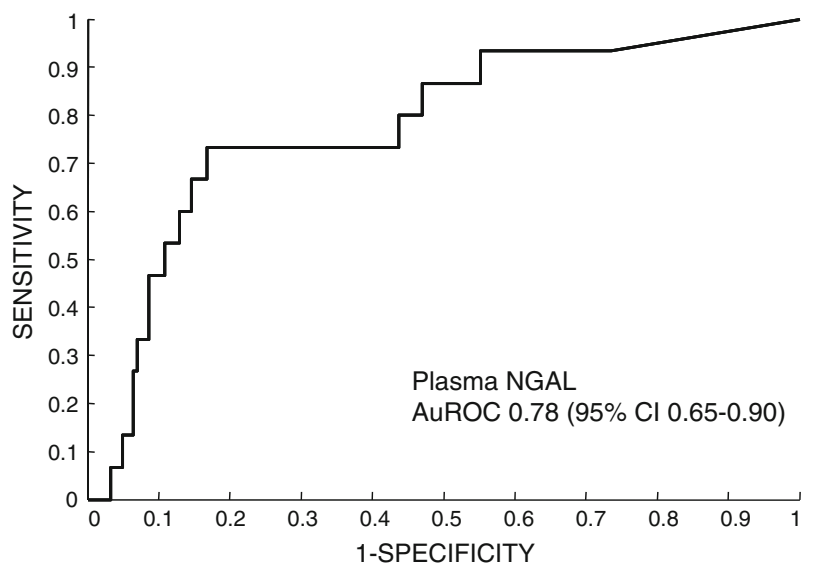

Fig. 3 Receiver operator characteristic (ROC) curve for plasma NGAL. The area under the ROC is 0.78 (95\% CI 0.65-0.90), demonstrating a good performance for the diagnosis of AKI within the next $48 \mathrm{~h}$

AKI development within the next $48 \mathrm{~h}$ from the first measurement (Fig. 3), with an area under ROC (AuROC) of 0.78 (95\% CI $0.65-0.90)$. Using a threshold value of $150 \mathrm{ng} / \mathrm{ml}$ for pNGAL, the sensitivity was $73 \%$, and specificity was $81 \%$ (Table 2). Plasma NGAL levels $>150 \mathrm{ng} / \mathrm{ml}$ were associated with increased odds of AKI of 11.8 (95\% CI 3.5-39.2). Furthermore, pNGAL was also a good predictor for RRT use during the ICU stay (AuROC 0.82, 95\% CI 0.70-0.95). However, its performance was comparatively weaker with regards to prediction of AKI occurring within the next 5 days, ICU mortality, and the composite endpoint of RRT and ICU mortality (AuROC 0.67, 0.67 and 0.68, respectively).

We also described the cohort with respect to the ascertained threshold pNGAL value (see ESM File 1, ESM Table 2). The findings are similar to Table 1 , reflecting the association between AKI and elevated pNGAL levels. Patients with first pNGAL $>150 \mathrm{ng} / \mathrm{ml}$ were older, were more likely to have CKD, have higher APACHE II and SOFA scores and SCr on ICU admission, and were more likely to have had exposure to potential nephrotoxins. Over $70 \%$ of these patients had AKI during their ICU stay. Also, this group had higher RRT use (11.7\%) and ICU mortality (27\%). The duration of AKI among survivors was longer in patients with first pNGAL $>150 \mathrm{ng} / \mathrm{ml}$ versus those with first
pNGAL $<150 \mathrm{ng} / \mathrm{ml}$ (4 days, IQR 2,7 vs. 2 days, IQR 1,6, respectively, $P=0.013$ ).

NGAL and severity of illness and AKI

All blood specimens were used to calculate peak and mean pNGAL values [4]. Peak pNGAL concentrations increased with worsening severity of AKI (Table 3). There was a similar relationship between mean pNGAL levels and RIFLE-max class (Non-AKI $71.4 \mathrm{ng} / \mathrm{ml}$, IQR 43.9, 124.3; Risk 139.9 ng/ml, IQR 67.4, 185.0; Injury $161.6 \mathrm{ng} / \mathrm{ml}, \mathrm{IQR} 106.9-286.1$; Failure $522.0 \mathrm{ng} / \mathrm{ml}$, IQR 288.2-1061.8; $P<0.001$ by Kruskal-Wallis test). Peak pNGAL levels also correlated well with RIFLE-max class $(R=0.554, P<0.001)$. Patients were further grouped according to APACHE II, SAPS II and SOFA scores, and by presence of sepsis. In general, pNGAL levels were higher among patients with higher severity of illness scores, whether expressed as peak pNGAL (Table 3), mean pNGAL or first pNGAL levels (data not shown). There were weak but highly statistically significant correlations between peak NGAL levels and APACHE II $(R=0.287, P<0.001)$, SAPS II $(R=0.215, P<0.001)$ and SOFA scores $(R=0.289, P<0.001)$. There was no significant difference in pNGAL among patients with and without sepsis or SIRS (Table 3).

Table 4 shows the results of posthoc univariate and multivariate logistic regression analysis using age, APACHE II scores and first pNGAL concentration as independent variables to predict AKI within $48 \mathrm{~h}$ of admission. Age was not significant in either the univariate or multivariate model, whereas APACHE II was marginally significant, and NGAL was highly significant. The adjusted odds ratio for pNGAL did not depend significantly on which ICU severity score was used (APACHE II, SAPS II or SOFA) in the model; the best model was that using the APACHE II.

\section{Discussion}

We conducted a prospective study to evaluate plasma NGAL for the early diagnosis of AKI in a heterogeneous

Table 2 Test characteristics of plasma NGAL for acute kidney injury and clinical outcomes

\begin{tabular}{llllll}
\hline & ROC analysis & \multicolumn{2}{l}{ At cutoff of $150 \mathrm{ng} / \mathrm{ml}$} & \\
\cline { 3 - 6 } & AuC ROC (95\% CI) & Sensitivity $(95 \% \mathrm{CI})$ & Specificity $(95 \% \mathrm{CI})$ & PPV (95\% CI) & NPV (95\% CI) \\
\hline AKI within 48 h & $0.78(0.65-0.90)$ & $0.73(0.45-0.92)$ & $0.81(0.75-0.86)$ & $0.24(0.13-0.39)$ & $0.97(0.93-0.99)$ \\
AKI within 5 days & $0.67(0.55-0.79)$ & $0.46(0.27-0.67)$ & $0.80(0.74-0.86)$ & $0.26(0.14-0.41)$ & $0.91(0.85-0.95)$ \\
RRT within ICU stay & $0.82(0.70-0.95)$ & $0.87(0.60-0.98)$ & $0.65(0.60-0.71)$ & $0.12(0.06-0.19)$ & $0.99(0.96-1.00)$ \\
ICU mortality & $0.67(0.58-0.77)$ & $0.60(0.45-0.73)$ & $0.68(0.61-0.73)$ & $0.28(0.20-0.37)$ & $0.89(0.84-0.93)$ \\
RRT + ICU mortality & $0.68(0.59-0.77)$ & $0.60(0.47-0.73)$ & $0.68(0.62-0.74)$ & $0.31(0.23-0.41)$ & $0.88(0.82-0.92)$ \\
\hline
\end{tabular}


Table 3 Peak plasma NGAL values by patient subgroups

\begin{tabular}{|c|c|c|c|}
\hline Category & Mean \pm SD & Median (IQR) & $P$ \\
\hline \multicolumn{4}{|l|}{ RIFLE-max } \\
\hline NonAKI & $162.1 \pm 302.8$ & $102.4(67.4,147.5)$ & \multirow[t]{4}{*}{$<0.001$} \\
\hline Risk & $229.6 \pm 199.4$ & $171.4(101.9,281.7)$ & \\
\hline Injury & $332.4 \pm 314.2$ & $214.9(137.5,419.5)$ & \\
\hline Failure & $803.8 \pm 529.2$ & $620.3(406.4,1366.4)$ & \\
\hline \multicolumn{4}{|c|}{ APACHE II } \\
\hline$\leq$ Median & $219.0 \pm 361.0$ & $117.4(77.9,193.9)$ & \multirow{2}{*}{$<0.001$} \\
\hline$>$ Median & $329.8 \pm 387.8$ & $173.0(89.4,407.8)$ & \\
\hline \multicolumn{4}{|l|}{ SAPS II } \\
\hline$\leq$ Median & $223.1 \pm 354.7$ & $123.4(76.0,224.8)$ & \multirow[t]{2}{*}{0.005} \\
\hline$>$ Median & $322.8 \pm 395.1$ & $152.8(90.2,395.4)$ & \\
\hline \multicolumn{4}{|l|}{ SOFA } \\
\hline$\leq$ Median & $248.1 \pm 390.5$ & $120.9(77.0,214.8)$ & \multirow[t]{2}{*}{0.001} \\
\hline$>$ Median & $310.5 \pm 352.1$ & $175.2(100.4,401.9)$ & \\
\hline \multicolumn{4}{|l|}{ Sepsis } \\
\hline No & $264.9 \pm 394.0$ & $133.2(80.0,244.4)$ & \multirow[t]{2}{*}{0.334} \\
\hline Yes & $281.2 \pm 350.2$ & $137.5(86.6,373.8)$ & \\
\hline \multicolumn{4}{|c|}{ Sepsis or SIRS } \\
\hline No & $265.6 \pm 335.7$ & $140.9(82.1,255.0)$ & \multirow[t]{2}{*}{0.717} \\
\hline Yes & $275.8 \pm 410.3$ & $132.2(79.2,283.3)$ & \\
\hline
\end{tabular}

Median APACHE II score $=18$

Median SAPS II score $=40$

Median SOFA score $=5$

adult population. The key findings of this study are the following: first, $44 \%$ of ICU patients had AKI during their ICU stay, and nearly $30 \%$ had this within $24 \mathrm{~h}$ of admission, consistent with prior studies [9]. Second, plasma NGAL levels are significantly higher in ICU patients compared to healthy adults and are higher in AKI patients compared to non-AKI patients. Third, pNGAL was a good independent predictor for the development of AKI within the next $48 \mathrm{~h}$ (AuROC 0.78, 95\% CI 0.650.90 ) and for RRT use during the ICU stay (AuROC 0.82, 95\% CI 0.70-0.95). Fourth, pNGAL correlated with severity of AKI, as well of severity of illness.

Human NGAL is a $25-\mathrm{kDa}$ protein covalently bound to gelatinase and is one of the most upregulated transcripts in the kidney very early after acute injury [1]. To our knowledge, this prospective study is the first to examine plasma NGAL in a heterogeneous adult population. The AuROC in our study is highly consistent with that reported in 19 studies across different AKI settings (Haase et al., AJKD 2009, in press), but slightly lower than that reported in pediatric studies [10-12].
This is related to multiple factors, including the presence of co-morbid conditions and underlying chronic kidney disease (CKD) in our adult ICU population compared to children. These conditions may lead to elevation of NGAL levels independent of renal injury, which could confound the predictive ability of pNGAL for AKI [13-15]. Moreover, unlike surgery or radiocontrast exposure, neither the timing nor severity of the renal insult is known in a general ICU. In the setting of cardiopulmonary bypass, for example, there is a temporally predictable injury to the kidneys, and surrogate markers of the severity of injury such as cross-clamp time are available. In contrast nephropathy, the amount of administered radiocontrast may be a surrogate for the degree of renal injury. In comparison, although pNGAL could be measured in critically ill patients within the first $24 \mathrm{~h}$ of admission to the ICU, this may not necessarily be the first $24 \mathrm{~h}$ of their disease process. Furthermore, in the medical ICU, the kidney is likely to receive "multiple hits" of varying severity, quite different from the seemingly "single hit" model encountered in most surgical situations.

Both serum and urine NGALs (uNGAL) have been studied as markers for AKI in critically ill pediatric patients. Serum NGAL was a highly sensitive but nonspecific predictor of AKI in children with septic shock (AuROC 0.68) [5]. In a heterogeneous pediatric ICU population, uNGAL was a useful early marker for development of AKI within 48 h, with AuROC 0.78 [4]. Similar to our own findings, mean and peak uNGAL increased with worse pediatric RIFLE class. They also observed a weak correlation between Pediatric Risk of Mortality (PRISM) scores and mean and peak UNGAL in AKI patients.

In adults, Makris et al. found UNGAL measured within $24 \mathrm{~h}$ from injury to be a useful early AKI marker in critically ill trauma patients, with AuROC of 0.97 [2]. This situation is analogous to that of surgery or contrast nephropathy, where the timing of renal insult is clearly defined. In addition, the AKI group was significantly older than the non-AKI group (median age 60 vs. 32 years, respectively), and this may have contributed to the relatively high AuROC in this study. In a recent singlecenter study in critically ill adults, uNGAL was shown to have moderate predictive utility for the development of AKI, although its additional contribution to conventional clinical risk factors appears limited [16].

Table 4 Univariate and multivariate analysis of plasma NGAL for prediction of acute kidney injury (AKI) within $48 \mathrm{~h}$

\begin{tabular}{lllll}
\hline & \multicolumn{2}{l}{ Univariate } & & \multicolumn{2}{l}{ Multivariate } \\
\cline { 2 - 3 } & OR $(95 \% \mathrm{CI})$ & $P$ & OR $(95 \% \mathrm{CI})$ & $P$ \\
\hline Age & $1.003(0.977-1.030)$ & 0.832 & $0.987(0.959-1.015)$ & 0.360 \\
APACHE II & $1.074(0.999-1.154)$ & 0.052 & $1.084(1.000-1.174)$ & 0.050 \\
$\log ($ pNGAL) & $4.989(1.496-5.652)$ & 0.002 & $3.059(1.509-6.201)$ & 0.002 \\
\hline
\end{tabular}

The odds ratio for each independent variable is a point estimate computed from the model coefficient (beta). The adjusted odds ratio for pNGAL did not depend significantly on which ICU severity score was used (APACHE II, SAPS II or SOFA) in the model; the best model was that using the APACHE II 
Our study makes an important contribution to the current body of literature on NGAL and AKI. Ours is the first study to prospectively evaluate plasma NGAL in all patients admitted to an adult general ICU, making the results more readily applicable to the usual ICU population. The cohort was followed through the entire ICU stay. This study also utilizes both the SCr and urine output criteria of RIFLE, as recommended by the ADQI group [6]. Moreover, we used a standardized user-friendly platform; a recent meta-analysis reported better AuROCs with standardized NGAL assays compared to "research-based" ELISA assays (Haase et al., AJKD 2009, in press). Our use of a commercially available platform is also a potential strength, since this would allow other investigators to confirm these findings. Our results show that pNGAL is a useful biomarker even in a population with common co-morbidities, such as diabetes, CKD and exposure to potential nephrotoxins. As such, it complements the original study that recruited a homogenous cohort of children who had no co-morbidities and had normal renal function [10]. Finally, we demonstrate in adult ICU patients that pNGAL correlates with severity of AKI and critical illness, yet remains an independent predictor for AKI after adjustment for severity of illness. Other investigators have looked at other novel biomarkers for AKI in critically ill adults, including cystatin-C [17], IL-18 [8], IL-6 [18], soluble TNF-receptors (S-TNF-R) [19], urinary $\mathrm{N}$-acetyl- $\beta$-(D)-glucominidase activity (NAG) [20, 21], kidney injury molecule-1 (KIM-1) [20, 21] and matrix metalloproteinase-9 (MMP-9) [21]. Some of these studies looked at either selected high-risk ICU populations [17] or enrollees in randomized controlled trials $[8,18,19]$; therefore, the results may not be broadly applicable to the more general population seen in clinical practice.

We acknowledge certain limitations in this study. This study enrolled patients from a single center; data from a multicenter study would be more generalizable. Moreover, nearly $30 \%$ of patients in this study already fulfilled criteria for AKI on admission to the ICU. This is consistent with data from a large observational study that showed that AKI occurred in $36 \%$ on the day of ICU admission [9]. This may be an important limitation for any studies on early biomarkers in an unselected adult ICU population.

In summary, plasma NGAL appears to be a useful early marker for the development of AKI in a large heterogeneous adult ICU population, allowing the diagnosis of AKI up to $48 \mathrm{~h}$ prior to a clinical diagnosis based on AKI consensus definitions. It is also a good predictor of need for RRT, and correlates with AKI severity and overall severity of illness. Early identification of patients who are likely to develop AKI and those who may require RRT will allow early targeted therapy of these high risk patients with potentially beneficial treatments before significant renal injury occurs.

Acknowledgments The authors are very grateful to Drs. Giampiero Gallo, Lusine Pogoshyan, Anton Verbine and the ICU physicians and staff, who were all critical to the project's implementation. This work was presented in part at the American Society of Nephrology 2008 meeting, held in Philadelphia, PA, USA. This work was made possible through the International Society of Nephrology-funded fellowship of Dr. Dinna Cruz. The authors also thank Ken Kupfer, Kristina Little, Irene Bolgan and Brian Noland for statistical and technical advice. The study was supported by a grant from the Associazione Amici del Rene Vicenza (AARVI), a non-profit private organization, and plasma NGAL measurement provided by Biosite, Inc. Neither AARVI nor Biosite had any role in study design, data analysis, data interpretation or writing of the report. Claudio Ronco had full access to all the data in the study and had final responsibility for the decision to submit for publication. Agreement to submit for publication was obtained from all authors.

Conflict of interest statement Drs. Cruz and Ronco have received an honorarium for speaking for Biosite Incorporated. All other authors declare that they have no conflict of interest.

Open Access This article is distributed under the terms of the Creative Commons Attribution Noncommercial License which permits any noncommercial use, distribution, and reproduction in any medium, provided the original author(s) and source are credited.

\section{References}

1. Devarajan P (2008) NGAL in acute kidney injury: from serendipity to utility. Am J Kidney Dis 52:395-399

2. Makris K, Markou N, Evodia E, Dimopoulou E, Drakopoulos I, Ntetsika K, Rizos D, Baltopoulos G, Haliassos A (2009) Urinary neutrophil gelatinaseassociated lipocalin (NGAL) as an early marker of acute kidney injury in critically ill multiple trauma patients. Clin Chem Lab Med 47:79-82
3. Hirsch R, Dent C, Pfriem H, Allen J, Beekman RH 3rd, Ma Q, Dastrala S, Bennett M, Mitsnefes M, Devarajan P (2007) NGAL is an early predictive biomarker of contrast-induced nephropathy in children. Pediatr Nephrol 22:2089-2095

4. Zappitelli M, Washburn KK, Arikan AA, Loftis L, Ma Q, Devarajan P, Parikh CR, Goldstein SL (2007) Urine neutrophil gelatinase-associated lipocalin is an early marker of acute kidney injury in critically ill children: a prospective cohort study. Crit Care 11:R84
5. Wheeler DS, Devarajan P, Ma Q, Harmon K, Monaco M, Cvijanovich N, Wong HR (2008) Serum neutrophil gelatinase-associated lipocalin (NGAL) as a marker of acute kidney injury in critically ill children with septic shock. Crit Care Med 36:1297-1303

6. Bellomo R, Ronco C, Kellum JA, Mehta RL, Palevsky P (2004) Acute renal failure-definition, outcome measures, animal models, fluid therapy and information technology needs: the Second International Consensus Conference of the Acute Dialysis Quality Initiative (ADQI) Group. Crit Care 8:R204-R212 
7. Levey AS, Bosch JP, Lewis JB, Greene T, Rogers N, Roth D (1999) A more accurate method to estimate glomerular filtration rate from serum creatinine: a new prediction equation. Modification of Diet in Renal Disease Study Group. Ann Intern Med 130:461-470

8. Parikh CR, Abraham E, Ancukiewicz M, Edelstein CL (2005) Urine IL-18 is an early diagnostic marker for acute kidney injury and predicts mortality in the intensive care unit. J Am Soc Nephrol 16:3046-3052

9. Bagshaw SM, George C, Bellomo R (2008) Early acute kidney injury and sepsis: a multicentre evaluation. Crit Care 12:R47

10. Mishra J, Dent C, Tarabishi R, Mitsnefes MM, Ma Q, Kelly C, Ruff SM, Zahedi K, Shao M, Bean J, Mori K, Barasch J, Devarajan P (2005) Neutrophil gelatinase-associated lipocalin (NGAL) as a biomarker for acute renal injury after cardiac surgery. Lancet 365:1231-1238

11. Bennett M, Dent CL, Ma Q, Dastrala S, Grenier F, Workman R, Syed H, Ali S, Barasch J, Devarajan P (2008) Urine NGAL predicts severity of acute kidney injury after cardiac surgery: a prospective study. Clin J Am Soc Nephrol 3:665-673

12. Dent CL, Ma Q, Dastrala S, Bennett M, Mitsnefes MM, Barasch J, Devarajan P (2007) Plasma neutrophil gelatinaseassociated lipocalin predicts acute kidney injury, morbidity and mortality after pediatric cardiac surgery: a prospective uncontrolled cohort study. Crit Care 11:R127
13. Malyszko J, Malyszko JS, Bachorzewska-Gajewska H, Poniatowski B, Dobrzycki S, Mysliwiec M (2009) Neutrophil gelatinase-associated lipocalin is a new and sensitive marker of kidney function in chronic kidney disease patients and renal allograft recipients. Transplant Proc 41:158-161

14. Bolignano D, Lacquaniti A, Coppolino G, Campo S, Arena A, Buemi M (2008) Neutrophil gelatinase-associated lipocalin reflects the severity of renal impairment in subjects affected by chronic kidney disease. Kidney Blood Press Res 31:255-258

15. Poniatowski B, Malyszko J, Bachorzewska-Gajewska H, Malyszko JS, Dobrzycki S (2009) Serum neutrophil gelatinase-associated lipocalin as a marker of renal function in patients with chronic heart failure and coronary artery disease. Kidney Blood Press Res 32:77-80

16. Siew ED, Ware LB, Gebretsadik T, Shintani A, Moons KG, Wickersham N, Bossert F, Ikizler TA (2009) Urine neutrophil gelatinase-associated lipocalin moderately predicts acute kidney injury in critically ill adults. J Am Soc Nephrol 20:1823-1832
17. Herget-Rosenthal S, Marggraf G, Husing J, Goring F, Pietruck F, Janssen O, Philipp T, Kribben A (2004) Early detection of acute renal failure by serum cystatin C. Kidney Int 66:1115-1122

18. Chawla LS, Seneff MG, Nelson DR, Williams M, Levy H, Kimmel PL, Macias WL (2007) Elevated plasma concentrations of IL-6 and elevated APACHE II score predict acute kidney injury in patients with severe sepsis. Clin J Am Soc Nephrol 2:22-30

19. Iglesias J, Marik PE, Levine JS (2003) Elevated serum levels of the type I and type II receptors for tumor necrosis factor-alpha as predictive factors for ARF in patients with septic shock. Am J Kidney Dis 41:62-75

20. Liangos O, Perianayagam MC, Vaidya VS, Han WK, Wald R, Tighiouart H, MacKinnon RW, Li L, Balakrishnan VS, Pereira BJ, Bonventre JV, Jaber BL (2007) Urinary N-acetyl-beta-(D)glucosaminidase activity and kidney injury molecule-1 level are associated with adverse outcomes in acute renal failure. J Am Soc Nephrol 18:904-912

21. Han WK, Waikar SS, Johnson A, Betensky RA, Dent CL, Devarajan P, Bonventre JV (2008) Urinary biomarkers in the early diagnosis of acute kidney injury. Kidney Int 73:863869 